\title{
Plasticized poly(lactic acid) with low molecular weight poly(ethylene glycol) : mechanical, thermal, and morphology properties
}

\begin{abstract}
Poly(lactic acid) PLA was plasticized with low molecular weight poly(ethylene glycol) PEG200 to improve the ductility of PLA, while maintaining the plasticizer content at maximum $10 \mathrm{wt} \%$. Low molecular weight of PEG enables increased miscibility with PLA and more efficient reduction of glass transition temperature $(\mathrm{Tg})$. This effect is enhanced not only by the low molecular weight but also by its higher content. The tensile properties demonstrated that the addition of PEG-200 to PLA led to an increase of elongation at break ( $>7000 \%)$, but a decrease of both tensile strength and tensile modulus. The plasticization of the PLA with PEG-200 effectively lowers Tg as well as cold-crystallization temperature, increasing with plasticizer content. SEM micrographs reveal plastic deformation and few long threads of a deformed material are discernible on the fracture surface. The use of low molecular weight PEG-200 reduces the intermolecular force and increases the mobility of the polymeric chains, thereby improving the flexibility and plastic deformation of PLA.
\end{abstract}

Keyword: Biodegradable; Blends; Plasticizer. 